\title{
Web-based Agricultural Technology Evaluation Information System: Design, Application and Evaluation
}

\author{
Yanzhao Ren ${ }^{1}$, Minjuan Wang ${ }^{1}$, Wanlin Gao 1*, Hui Hu1, Xuerui Chen1 \\ ${ }^{1}$ College of Information and Electrical Engineering, China Agricultural University, 100083, Beijing, China;
}

\begin{abstract}
This study describes the web-based agricultural technology evaluation information system, which has been shown to provide a flexible interface with which to arrange various assessment and information management function procedures. Secondly, this study examines the effects of the application of the academic misconduct detection mechanism and abnormally scoring mechanism. In an evaluative study with the two evaluation business cycle of the system, it has been found that this system is more efficient, more accurate and more rigorous than the traditional offline evaluation model. It has also been shown that cheating behaviour in agricultural technological achievements information declaration and evaluation link can be detected accurately and promptly.
\end{abstract}

\section{INTRODUCTION}

Agricultural science and technology achievements transformation is the only way to improve technological productivity and promote agriculture. In this process, the protection of the achievements' intellectual property rights is very important ( $\mathrm{Li}$ xian, 2006). In the past few years, our government has set up special funds and awards at all levels to support these types of transformation projects, which achieved good results. However, some important questions arise (Li HaiYan, 2000). Is it possible to keep fair and open in the process of agricultural science and technology achievements evaluation? How should the reviews of these achievements be standardized? How should the achievements be managed and promoted? These are the problems we need to study in the process of agricultural scientific and technological achievements evaluation (M. Radwan, Senousy, \& M. Riad, 2017) (Jin ZhenHui, 2006).

Some people have done some works on the management of evaluation of scientific and technological achievements. Yemao and others designed the Dayu Water Conservancy Science Information System, which is the basis of the Dayu prize award (Yang SiBo, 2002) (Ye Ma, 2007). Feng Li designed the Textile Science Appraisal System, which is divided into two parts, the back and the front. The background management functions include code, importing recommendation, and summary tables. The front include export and import of the recommendation, print recommendation, adding projects, submitting the recommendation online, etc. (Feng Li, 2012) (Wang ChenYe, 2012). Hua lei designed a model household agricultural science and technology application system, which can satisfy the declaration on the function, evaluation, examination and approval, and model household information management's needs (Hua Lei, 2013). The management system of scientific achievements main functions are to management the scientific research in colleges and universities, so as to help teachers report their scientific research and the staff in technology department to create statistics for everyone's scientific research, and provide the most direct basis for the bonuses(Huang Wei,2013)(Jiang LiHua,2014). However, there is no correlative literature for instituting a system for scientific and technological achievements reward system which can complete the entire workflow of Achievements evaluation on-line (Chen, 2017).

Based on the above analysis, we designed an agricultural science and technology achievements system based on the MVC (Model View Controller)(yurong,2013), using Spring MVC + Spring + Hibernate framework, which can managed organically and applied to evaluate the achievements(Vabo, Slettebø, $\&$ Fossum,2016). The goal of this system is to monitor the achievements online, in order to review and unify management of the business process and effectively improve and manage personnel's work efficiency. 


\section{ANALYSIS OF TECHNOLOGICAL ACHIEVEMENTS PROCESS}

Agricultural science and technology achievements assessing work process mainly includes achievement collection and review, expert estimation and results announcement. In the collection process, the information related to technique collection scope, time axis and sponsor is publicized at first. The qualified units apply and obtain the production application quota after they got the notice. And then those units distribute the application places to the achievements owners according to the rules of each unit. The holders who get the position prepare relative technical information and sent them to the qualified units. The units concentrate all the achievements files and sent them to sponsor. The sponsor and the qualified units all have rights to investigate and return the application documents. In the expert estimation link, technological subjects and reviewers are grouped according to professional field, and the judges participate in review meeting to grade fruitions after received an invitation. During this process, the organizer is responsible for supervising the reviewing and critiquing. After the estimation work, the results are checked and publicized. In traditional way, the management of activities associate with agricultural scientific and technological achievements was carried out with noninformatization (Theo S.Eicher, 2017) (Yuan Xiaoqing, 2015). The information of the achievements are transported in the form of paper material and accessory disk. As a result, the processing procedure is relatively cumbersome and the management sites are scattered, which makes mistakes and the loss of the applying materials a common occurrence (Lingxian Zhang2013). Decentralized management creates information synchronization, delayed feedback of consequences, low efficiency of management, low management quality, and unorganized data. Much more resources are spent on unifying progress of each link, organizing on-the-spot meeting for evaluation and so on.

In this paper, the way to complete the whole process steps in the evaluation online, beginning with the quota distribution and ending with review completed, is researched (Xue Dong,2007). And the technic of academic misconduct detection and monitoring of abnormally scoring. This chain of processing steps are two of the most important and complicated process segments in the review workflow (Hou Xin, 2015).

\section{DEIGN OF KEY TECHNOLOGY ROADMAP}

As mentioned above, the functions that detect the academic misconduct and monitor the abnormally scoring are foundation for ensuring the fairness during system running process.

\subsection{Academic misconduct detection mechanism}

The academic immoral behaviours in this activity mainly include the following three cases: the award-winning scientific and technological achievements participate in the evaluation again, the core technology of the achievement is similar to other people, and the same scientific and technological achievements occupies more than one quota. If one achievement fit into one of these scenarios, the contents of the accomplishment introduction and technical innovation of it will have one corresponding version which has a high repetition rate at least.

Aiming at the above problems, this paper presents two kinds of content comparison models: whole database comparison and specify results comparison. And the two ways mentioned above have the same content comparison logic.

In this algorithm, the segmentation of the literatures being compared will be finished at first. Before the next step, an important parameter which limits the conditions for repetitive content should be determined. If the number of continuous character which are continuous character with others is equal or more than the figure be set before, these character segment are firmly believed to be consistent with repeated conditions. The matrix can be used to explain this process.

$S_{i}=\left[\begin{array}{c}i \\ \text { detectsentencetxt } \\ \text { simsentencetxtname } \\ \text { simsentencetxt } \\ \text { simsentencestate } \\ \text { beginindexes }[0] \\ \text { beginindexes }[1] \\ \text { endindexes }[0] \\ \text { endindexes }[1]\end{array}\right]^{T}$

The elements of this matrix record the comparison results between single sentences. In this matrix, ' $i$ ' means the sentence label of the reference articles, and other parameters have the following mapping relationships: 'detectsentencetxt', the content of detected sentence; 'simsentencetxtname', reference name; 'simsentencetxt', the content of referenced sentence; 'simsentencestate', marking of test results; 'beginindexes[0]', the starting location of repeated section in detected sentence; 'endindexes[0]', the ending location of repeated section in detected sentence; 'beginindexes[1]', the starting location of repeated section in referenced sentence; 'endindexes[1]', the ending location of repeated section in referenced sentence.

$$
P=\left[\begin{array}{llll}
\text { detectsentencetxt } & S_{1} & \cdots & S_{i}
\end{array}\right]
$$

The data about the result that one sentence compared with all sentences of one achievement's content is included in $\mathrm{P}$. The meaning of parameter $\mathrm{i}$ is the number of sentences of the whole reference article. 


$$
L=\left[\begin{array}{llll}
\text { detectsentencetxt } \mid & P_{1} & \cdots & P_{m}
\end{array}\right]
$$

In this way, the $\mathrm{L}$ can include the results of the comparison between the sentences with the achievements which are pre-set. The index $\mathrm{m}$ means the number of articles which are specified contrast samples.

$$
R=\left[\begin{array}{cc}
1 & L_{1} \\
\cdots & \cdots \\
n \mid & L_{n}
\end{array}\right]
$$

The index $\mathrm{i}$ is the number of the sentences of the detected achievement's contents. And the label of sentence is put in $\mathrm{R}$ with the result matrix $\mathrm{L}$ which is mapped with it. By this mode, we encapsulate the multidimensional test results into a matrix and it can be reversed to the bottom.

\subsection{Abnormally scoring mechanism}

The organizational form of the assessing activity is many experts to many projects. The judges and achievements are grouped on the basis of their profession. In assessment, the scoring mechanism will maintain fairness with two modes: weighting method and re-examining method. And the precondition of monitoring core algorithm of them is that the scoring of experts is in accord with the independent and identically distributed. This rule can be obtained by analyzing historical data (João Carneiro, Diogo Martinho, Goreti Marreiros, 2016). After the first round of evaluation, we can get the expectation $\left(\mu_{\mathrm{p}}\right)$ and the standard deviation $\left(\sigma_{\mathrm{p}}\right)$ of the scores of every projects. So if the score given by the judges is in the range of $\mu_{\mathrm{p}} \pm \sigma_{\mathrm{p}}$, the review behaviour of them is concealed normal and the weight (w) of score will be set to 1 . If the score is in the range of $\mu_{p} \pm \sigma_{p}$ to $\mu \mathrm{p} \pm 2 \sigma \mathrm{p}$, the weight should be set to 0.5 for it is believed lower than the norms. The weight is 0 for the residual condition. The algorithm of the final score $\left(s_{p}\right)$ is as follows:

$$
s_{p}=\frac{\sum_{j=1}^{m} S_{j} * \mathcal{W}_{j}}{\sum_{i=1}^{m} \mathcal{W}_{i}}
$$

In the above formula, $\mathrm{m}$ is the number of experts who evaluate the project. The factor $S_{j}$ is the score of one project from an expert and $\mathrm{S}_{\mathrm{j}}$ is the corresponding weight. If the most of the weight indexes of the scores which are given by one expert is unreasonably low, the result will be investigated. As corresponding measures, new judges will be attended to re-examine the relative projects.

May be parts of members of one group will give a low label grade to an achievement, and this will lead to a low s. It should be also be paid attention to. Solving this problem, the scores $\left(s_{p}\right)$ of the entire group are taken as sample and the $\mu_{\mathrm{g}}$ and $\sigma_{\mathrm{g}}$ are gotten. So if the $\mathrm{s}_{\mathrm{p}}$ is not in the range of $\mu \mathrm{g} \pm 2 \sigma \mathrm{g}$, the evaluation behaviour point at it will be assessed and whether it should be re-rated would be decided according to the assessment result.

\section{SYSTEM DEVELOPMENT}

\subsection{System service}

The system in general needs to implement the following functions:

(1) Reward for users to declare an index, the results of the review and distribution, and the distribution of the unified management of achievements.

(2) Online users declare a function of agricultural scitech achievements.

(3) Recommendation units to the agricultural scientific and technological achievements for the management function.

(4) Evaluator review the user allocation of agricultural science and technology achievements score function.

Therefore, the system function modules are divided into different user login modules including, declared units, recommended unit for function module, review function module, and reward function module five parts. This module is shown in Figure 1.

\begin{tabular}{|c|c|c|l|l|}
\hline \multicolumn{5}{|c|}{ Online assessment system for agriculture technology } \\
\hline \multicolumn{3}{|c|}{ Business role } & Service role \\
\hline Host unit & Evaluator & $\begin{array}{c}\text { Recommendation } \\
\text { Unit }\end{array}$ & Applicant & System administrator \\
\hline
\end{tabular}

Figure 1 System Function Modules

Agricultural science and technology achievements of online return and review work process mainly includes initialization, confirmation, material declaration, opinion and order form review, distribution, Evaluator, expert ratings, and reviews.

This process is shown in figure 2 .

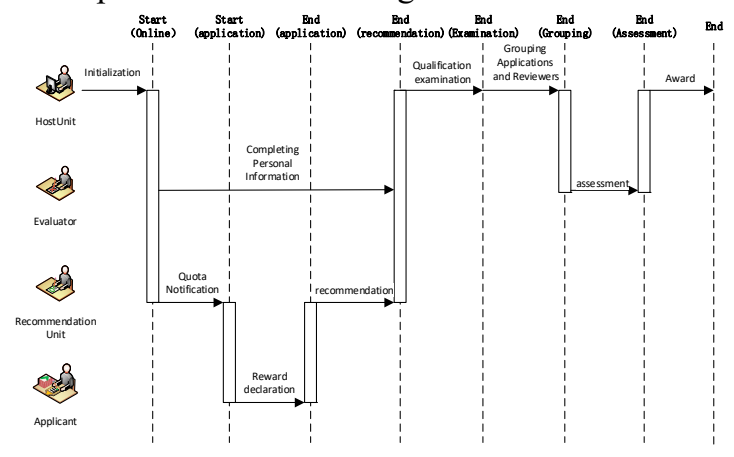

Figure 2 declare and review of flow diagram

According to the working process of the awards, the overall business process of the system is shown in figure 3(Saif Ur Rehman Khan, 2016).

(1) Reward for users to recommend unit assigned to declare index.

(2) Recommended unit in offline notification to declare indexes corresponding to user accounts about informing declare units. 
(3) Declare units begin after landing system online declaration, after the declaration is submitted to the archive.

(4) Recommended unit declares an initial review of scientific and technological achievements, if it is qualified to confirm recommend. If not qualified, then it is returned to the declare unit for further modification.

(5) Demand rewards have been recommended to the management of the group of the agricultural scientific and technological achievements. They are mainly a recommended form of rechecking during the course of agricultural scientific and technological achievements and review. The review form is done by the group for the work assigned, corresponding to review and Evaluator and if it is unqualified, they can choose to return for modification or have it directly eliminated. Directly eliminated results will not be sent back to participate in additional review.

(6) Evaluator can be assigned to their results in accordance with the scores and submit those results to be reviewed against the rules.

(7) It is expected for all the experts all items to make a review after comparison and appraisal, according to the rules, in order to select the corresponding project award.

(8) The results can be managed after the end of the link of prizes.

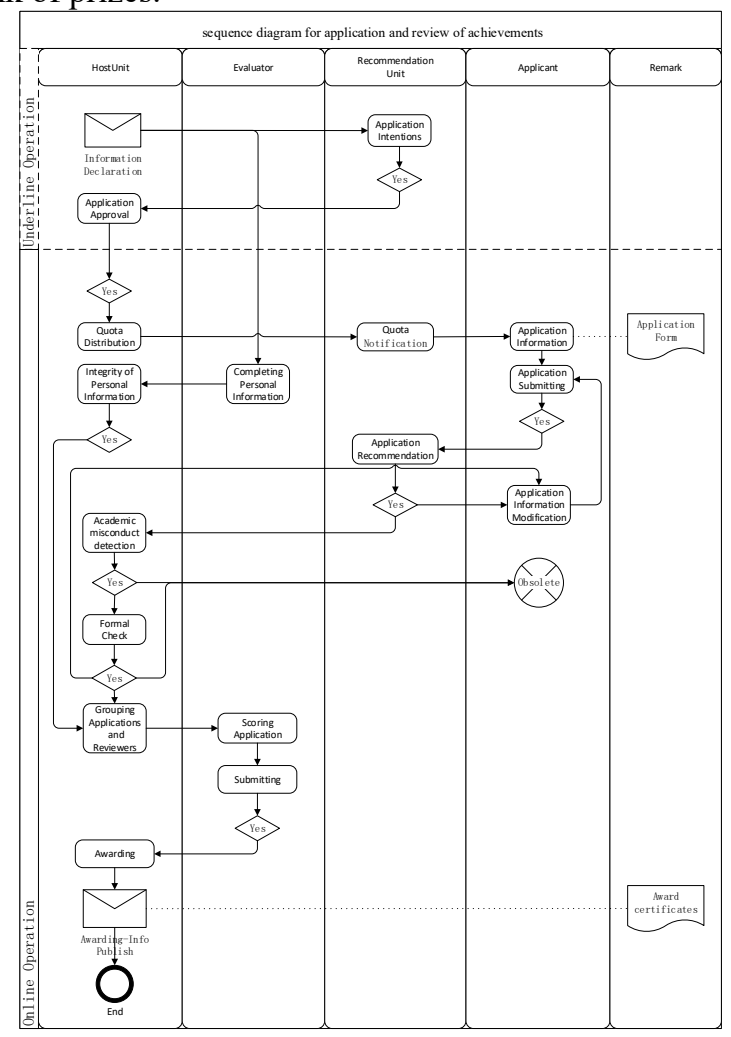

Figure 3. Overall business flow diagram

\subsection{System structure}

The platform is based on $\mathrm{B} / \mathrm{S}$ architecture, which both takes advantage of the network and the function of the system at the same time (Re Zhongfang, 2004). It adds the business logic layer and separates the user from the data layer, which reduces the load of the Web Server and avoids any negative influence caused by the overall performance of the whole system (Shi Xingjian, 2004). The System architecture (MVC) is shown in Figure4.

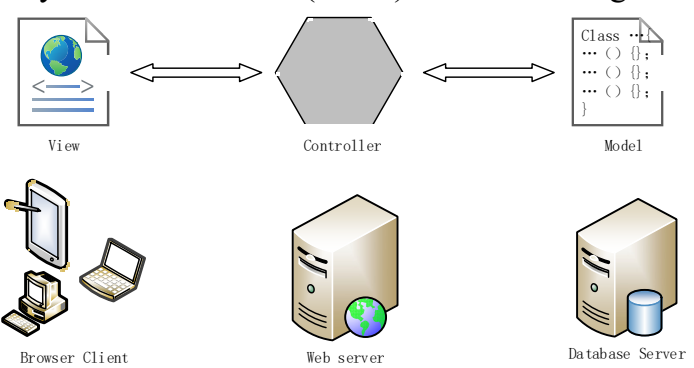

Figure 4 System architecture

The MVC model, with the characteristics of clear design, easy to use and extend distribution, separate the program performance, control and data, which has the advantage in a web application and in adaptation into the systems that are multiuser, extensible, maintainable, and have a high level of interactivity (J. C. Chang, S. R. Hanna,2004).

The presentation layer: The user interface section, used for the dialogue between the user and the system function. Mainly used to check the user keyboard input and the output of the operation result. To complete this, the languages JavaScript, jQuery were used (Xiaochang Liu, 2016).

The business logic layer: The business logic algorithm processes the input, called the database layer, and then returns the data results. The layer mainly includes the business functions and the data function, business function layer can be resolved within the function, but data function must rely on interactions with the data layer can be completed. And the subsystem in this part was developed the java language (Jie Shen, 2015).

The database layer: The centralized management of the database, including business entities and the data operation (Ximing Li, Jihong Ouyang, You Lu, 2015).

\section{SYSTEM APPLICATION}

The system is deployed on a server connected to Internet, so it can be tested by the users in different regions with different ways of surfing the Internet. The server's main configuration parameters are as follows: operating system: windows server 2008; web server: Tomcat 6.0; CPU: Xeon E3-1220; Memory type: 8G DDR3 ECC.

During the trial operation of the system, about 20 recommended units and 60 application unit or applicants were invited to load this system to complete the simulated reporting procedures. The system implements the reservation functions. In formal application, about 132 recommended units and 574 application units have use the system to participate in the information of their technological achievements.

\subsection{Duplicate checking function}

This function is evaluated with one case by comparing the detected achievement information with that of the previous award winning results. There are about 800 
samples and everyone has about 4000-5000 characters. The function is mainly directed against individual unethical behaviour. The similarity of every achievement can be queried by formulating factors. And the interface of the detailed information of repetition rate detection results is also be provided for every projects. It will lead to the similarity details page. In this page, if one or more sentences repeat with the sample, it will be shown in red. The related sentences, topic and award information of sample are also displayed.

\subsection{Abnormally scoring function}

To guarantee fairness of evaluation and validate the consistency of the evaluating result and Technical level of achievement, we prepare two algorithms described above. The scenario represents different angle of the measurement with group sample synthesis model.

In the details information analysis, we present scoring record for every projects. It is defined to a sufficient level of detail for investigating for the score of every metric given by experts. The same rule applies when the scoring behaviour of one expert.

\section{RESULTS AND DISCUSSION}

Compared with the traditional evaluation model and method, this system has the following features:

(1) The System Work Flow is clear.

For the characters of science achievements include systematic structure, correlation of discipline, the complexity of the content and species diversity, the achievements of agricultural science and technology of online reporting and review process has carried on the detailed analysis, fully reflecting the project application and review process, in order to declare the project appraisal status has carried on the detailed classification of users at a glance and is easy to operate. It can be found that the average expenditure of web-based system is considerably lower than that of traditional way for peer assessment cycle. And it also show that the average expenditure of second online cycle is less than the first one for the reason that part of the workload is spent on real time consulting services for system usage during the first on-line operation. The related time data are shown in table 1

\begin{tabular}{|c|c|c|c|c|c|}
\hline & \multicolumn{3}{|c|}{ traditional } & \multicolumn{2}{|c|}{ online } \\
\hline & $\begin{array}{l}2008- \\
2009\end{array}$ & $\begin{array}{l}2010- \\
2011\end{array}$ & $\begin{array}{l}2012- \\
2013 \\
\end{array}$ & $\begin{array}{l}2014- \\
2015 \\
\end{array}$ & $\begin{array}{l}2016- \\
2017 \\
\end{array}$ \\
\hline total $($ term $)$ & 355 & 367 & 373 & 587 & 769 \\
\hline $\begin{array}{l}\text { working hours } \\
\text { (h) }\end{array}$ & 2900 & 3110 & 3350 & 1140 & 970 \\
\hline $\begin{array}{l}\text { Average } \\
\text { expenditure } \\
\text { (h/term) }\end{array}$ & 8.17 & 8.47 & 8.98 & 1.94 & 1.39 \\
\hline
\end{tabular}

(2) Monitoring of academic wrongdoing

During the application submitting link, after the application units upload their application documents, the main technical content is extracted and stored in the database and it will be compared with the data in the historical database which includes the filing of scientific and technological achievements over the years. The result will generate a report contains repeat rate and duplicate content. These functions will prevent repeated declaration and plagiarism. During the first review cycle, there were 28 technical achievements were returned for content reasons. It is accounting for $5.6 \%$ of the total number of declarations. In the second cycle, it dropped to $0.1 \%$ with the same standard, it means there were only 1 of 769 were return.

(3) Supervising appraisal activity anomalies

To determine whether judges performed more objectively in their assessments of agriculture achievements? This system analysis review activity from two viewpoints, one is counting the total score and individual score of each project, then screening of abnormal data depend on the rating distribution. The other aspect is doing statistical analysis on judges' scoring data, emphasizing the unusual discrete case of per expert marking moths. After the first system cycle was end, we compared the assessment data from two aspects mentioned above. The lower the technological innovation was, the smaller the deviation was between the experts scoring, and therefore the more objective the score was. By using the system algorithm to detect the distribution of points can make the discrete fraction distribution is found in time. This function has played a good role, making the appeal rate drop year by year as shown in table 2 .

\begin{tabular}{cccccc}
\multicolumn{8}{c}{ Table2 Appeal rate } \\
\hline & \multicolumn{3}{c}{ traditional } & \multicolumn{2}{c}{ online } \\
& $2008-$ & $2010-$ & $2012-$ & $2014-$ & $2016-$ \\
& 2009 & 2011 & 2013 & 2015 & 2017 \\
\hline $\begin{array}{c}\text { appeal } \\
\text { rate }\end{array}$ & 12 & 10 & 13 & 1 & 0.3 \\
\hline
\end{tabular}

\section{CONCLUSION}

Peer-assessments are methods of evaluation highly recommended by researchers, but the traditional pen-andpaper-based peer-assessment method has many restrictions. This study contributes to the field by designing the web-based assessment information system, and provides the procedures for online information management, online review and academic wrongdoing monitoring expecting that it can help minimize the difficulties in agriculture technology information management and evaluation. With the network application review and most of the information becomes electronic, there will be a reduced volume of materials submitted. We expect that the web-based system will stimulate more in-depth thinking as well as further development on ways to integrate web technology with the agricultural technology assessment models. 


\section{ACKNOWLEDGEMENT}

This work was supported by National Spark Program Key Project (Grant \#: 2015GA600002).

\section{Authors' Instructions}

Yanzhao Ren, PhD Student, Box 105, China Agricultural University, No 17, QingHua East Road Haidian District, Beijing, P.R. China. Yanzhao Ren obtained his Master of Engineering in Agricultural Machine Engineering from Shandong University of Technology, Zibo, China in 2012.He is currently pursuing his $\mathrm{PhD}$ in Agricultural Electrification and Automation at China Agricultural University, Beijing. His research direction is computer application technology and intelligent information processing. His research subjects includes automatic control technology of greenhouse environment, automatic operating equipment, agricultural informatization, Agricultural IoT.

\section{References}

1. Chen, R. Y. (2017). An intelligent value streambased approach to collaboration of food traceability cyber physical system by fog computing. Food Control, 71, 124-136

2. M. Radwan, N., Senousy, M. B., \& M. Riad, A. E. D. (2017). A new expert system for learning management systems evaluation based on neutrosophic sets. Expert Systems

3. Vabo, G., Slettebø, Å. \& Fossum, M. (2016). An Evaluation of an Action Research Nursing Documentation Project. Journal of Clinical Nursing

4. Li Xian. (2006)Life Cycle Assessment Theory and System Analysis of Agricultural Science and Technology Achievements. Jilin University

5. Li HaiYan. (2000). Establishment of Index System for Medical Achievements and Development of Comprehensive Evaluation System. First Military Medical University

6. Yang SiBo. (2002). the evaluation method research and software system implementation of scientific and technical achievements [D]. Tianjin Polytechnic University.

7. Wang ChenYe. (2012). A Study on the Operation Mode and Performance Evaluation of Scientific and Technological Achievements in Universities of Our Country. Harbin Engineering University

8. Huang Wei. (2013). the Study on Performance Evaluation of China Scientific and Technological Achievement Transformation and its Influence Factors and Countermeasures. Jilin University,

9. Jiang LiHua. (2014). Study on Theory and Method of Science and Technology Innovation for Agriculture Institutes, Chinese Academy of Agricultural Sciences.

10. Ye Ma, Zhao Hui, Wang Lei, Li Jianghua. (2007). the design and implementation of Dayu water conservancy science information system. TECHNICAL SUPERVISION IN WATER RESOURCES, 15(5):49-51.

11. Hua Lei. (2013). the design and implementation of Agricultural science and technology system.

12. João Carneiro, Diogo Martinho, Goreti Marreiros, Paulo Novais. (2016). Intelligent negotiation model for ubiquitous group decision scenarios. Frontiers of Information Technology \& Electronic Engineering, 17(4): 296-308. http://dx.doi.org/10.1631/FITEE.1500344

13. Xue Dong-Liang. (2007). scientific research management system, AGRICULTURE NETWORK INFORMATION, (10):210-212.

14. Shi Xingjian, Xu Liangxian. (2004). Research and Application of Hibernate in Struts, COMPUTER ENGINEERING, 30(zl):165-167,190.

15. Feng Li. (2012). The development and application of textile science appraisal system.

16. Yuan Xiaoqing. (2015). Study on Model and Method of Agricultural Informatization Level and Benefit Evaluation. China Agricultural University.

17. Hou Xin. (2015). the Research on Quality Assurance and Quality Evaluation Mode of Postgraduate Education. Shanxi University of Finance and Economics.

18. Theo S.Eicher, OliyerRoehn. (2007). Sources of the German Productivity Demise: Tracing the Effects of Industry-Level Information and Communication Technology Investment. German Economic Review. (2)

19. Re Zhongfang, ZHANG Hua, YAN Ming-song, Chen Shifu. (2004). Overview of the Research in Model-View-Controller Pattern, APPLICATION RESEARCH OF COMPUTERS. 21(10).

20. Lingxian Zhang,Xue Liu,Daoliang Li,Zetian Fu.(2013).Evaluation of the rural informatization level in four Chinese regions: A methodology based on catastrophe theory. Mathematical and Computer Modelling. (3-4).

21. Jin ZhenHui. (2006). Research on the Method at Science and Technology Protect Evalution, Kunming University of Science and Technology.

22. J. C. Chang,S. R. Hanna.(2004).Air quality model performance evaluation. Meteorology and Atmospheric Physics, (1-3)

23. Xi-ming Li, Ji-hong Ouyang, You Lu. (2015). Topic modeling for large-scale text data. Frontiers of Information Technology \& Electronic Engineering, 16(6): 457-465. http://dx.doi.org/10.1631/FITEE.1400352

24. Saif Ur Rehman Khan, Sai Peck Lee, Mohammad Dabbagh, Muhammad Tahir, Muzafar Khan, Muhammad Arif. RePizer. (2016).a framework for prioritization of software requirements. Frontiers of Information Technology \& Electronic Engineering, 17(8): $750-765$. 